\title{
Fonction de la Croissance de Korf
}

\section{Richard Zarnovican}

\author{
Service canadien des Forêts \\ Centre de Recherches forestières des Laurentides \\ C. P. 3800 , Sainte-Foy, Québec
}

\section{Résumé}

Un bref examen de la formulation mathématique de la fonction de croissance de Korf a été effectué. On a examiné l'application de cette fonction dans les travaux dendrométriques et on a utilisé cette fonction de Korf pour étudier la relation hauteur en fonction de l'âge en prenant comme exemple l'épinette noire de trois sites différents.

\section{Abstract}

Korf's mathematical formula, three-parameter-growth function, was briefly analyzed. How this formula can be applied to forest mensuration was also examined. Korf's formula was used to study the relation between height/age for three black spruce stands.

\section{Introduction}

En 1939, V. Korf' a proposé une formulation mathématique particulière de la croissance des arbres et des peuplememts forestiers dans le temps. Elle a été élaborée depuis, dans ses travaux récents (Korf 1967, 1974) notamment grâce aux outils de calculs plus puissants.

II a paru intéressant: 1) d'examiner la fonction de Korf quant à sa formulation mathématique, 2) d'analyser son application forestière et finalement, 3) de l'appliquer à l'étude de la hauteur de l'épinette noire en fonction de l'âge sur trois sites de qualité différente.

\section{Formulation mathématique de la fonction de Korf}

Considérons que toute grandeur peut évoluer dans le temps d'après la fonction suivante:

$$
y=h(t)
$$

Notre intérêt, pour cette fonction, se limite seulement aux valeurs positives de $t$ et de $h$. La fonction (1) peut être considérée comme la solution d'une équation différentielle du premier ordre, à savoir

$$
H\left(t, y, y^{\prime}\right)=0
$$

où $y^{\prime}=\frac{d y}{d t}$. Considérons le taux de croissance $\alpha$ d'un élément de notre population,

$$
\alpha=\frac{h^{\prime}}{h}=\frac{1}{h} \frac{d h}{d t}
$$

II est naturel de penser que ce taux de croissance sera une fonction décroissante du temps et qu'il tendra asymptotiquement vers zéro. Korf a tenté de traduire cette hypothèse par l'équation très simple:

$$
\alpha=\frac{h^{\prime}}{h}=\frac{k}{t^{n}}, k>0, n>1
$$

Cette équation différentielle très simple s'intègre facilement et on obtient

$$
h(t)=A e^{\frac{k}{1-n} t^{n-1}}
$$

L'expression (5) est la fonction de croissance de Korf, dont on peut trouver un exemple typique à la figure 1. La courbe a l'allure d'un $S$ et possède un point d'inflexion pour

$$
t=\left(\frac{k}{n}\right)^{\frac{1}{n-1}} \text {; elle tend asymptotiquement vers } A \text {, }
$$

Korf, v. 1939. Prispevek k matematicke definici vzrustoveho zakona hmot lesnich porostu. Lesnicka pracr, p. 339-379. quand $t$ tend vers l'infini. Dans l'intervalle $\left(0,\left(\frac{k}{n}\right)^{\frac{1}{n-1}}\right)$ elle est convexe, et son point d'inflexion I se trouve au temps

$$
t_{1}=\left(\frac{k}{n}\right)^{\frac{1}{n-1}} \text { de coordonnées } I=\left[\left(\frac{k}{n}\right)^{\frac{1}{n-1}}, A \text { e } \frac{n}{n-1}\right] .
$$

La courbe devient concave dans l'intervalle

$\left(\left(\frac{k}{n}\right)^{\frac{1}{n-1}}, \infty\right)$ avec l'asymptote $y=A$, (fig. 1).

La fonction de Korf (5) est parfaitement définie par trois paramètres, à savoir $A, n$ et $k$. A partir des coordonnées du point d'inflexion de la courbe, il est facile d'établir que:

a) pour $n$ et $k$ constants, la valeur de l'asymptote est donnée par A et l'abscisse du point d'inflexion reste constante,

b) pour $n$ et $A$ constants, l'ordonnée du point d'inflexion reste constante,

c) finalement pour $k$ et $A$ constants, soit $I_{1}=\left(t_{1}, y_{1}\right)$ et $I_{2}=$ $\left(t_{2}, y_{2}\right)$ deux points d'inflexion, pour $n_{2}>n_{1}$ : on a alors $t_{2}<$ $t_{1}$ et $y_{2}>y_{1}$.

Les paramètres $n, k$ définissent la position du point d'inflexion (et non de l'asymptote) tandis que le paramètre $A$ définit la position de l'asymptote (et non du point d'inflexion).

\section{L'application de la fonction de Korf en dendrométrie}

Comme nous l'avons dit plus haut, l'équation de Korf (5) a été définie par l'intégration de l'équation différentielle (4), où $\alpha$ correspond au taux de croissance. Si on multiplie $\alpha$ par cent, on obtient le taux d'accroissement en pourcentage, indice très intéressant en dendrométrie.

$$
\begin{aligned}
& \text { La première dérivée de l'équation (5) } \\
& \begin{aligned}
\mathrm{v}(\mathrm{t}) & =\mathrm{y}^{\prime}=\frac{\mathrm{dy}}{\mathrm{dt}}=\mathrm{A} \mathrm{e}^{\frac{\mathrm{k}}{(1-\mathrm{n}) \mathrm{t}^{\mathrm{n}-1}}} \frac{\mathrm{k}}{\mathrm{t}^{\mathrm{n}}}= \\
& =\mathrm{A} \mathrm{e}^{\Phi(\mathrm{t})} \Phi^{\prime}(\mathrm{t})=\mathrm{A} \mathrm{e}^{\Phi(\mathrm{t})} \alpha
\end{aligned}
\end{aligned}
$$

nous permet d'établir la fonction de l'accroissement courant et elle correspond à la vitesse de croissance. La courbe v(t) (fig. 1) est asymétrique avec le maximum au temps $t_{1}=\left(\frac{k}{n}\right)^{\frac{1}{n-1}}$ et les deux points d'inflexion se trouvent aux temps $t_{3}$ et $t_{4}$ (fig. 1)

$$
\begin{gathered}
t_{3}=\left[\frac{3 n+\left(5 n^{2}-4 n\right)^{\frac{1}{2}}}{2 k}\right]^{\frac{1}{1-n}} ; \\
t_{4}=\left[\frac{3 n-\left(5 n^{2}-4 n\right)^{\frac{1}{2}}}{2 k}\right]^{\frac{1}{1-n}}
\end{gathered}
$$

et l'asymptote est l'axe de $t$

$$
h\left(0^{+}\right)=\lim _{t \rightarrow 0^{+}} \frac{k}{e^{(1-n) t^{n-1}}}=0
$$


II convient de rappeler que la courbe de l'accroissement courant peur être divisée d'après l'âge en segments qui correspondent dans la vie d'un arbre ou d'un peuplement aux phases de croissance.

Ainsi (figure 1), on peut parler de la phase juvénile pour le segment de la courbe $v(t)$ entre l'âge $0^{+}$et $t_{3}$, le segment de la même courbe compris entre le temps $t_{3}$ et $t_{4}$ représente la phase de la pleine vigueur, et finalement le segment de cette même courbe à droite de temps $t_{4}$ représente la phase de la sénescence.

L'intérêt de l'établissement de la courbe de l'accroissement courant et son temps de culmination pour les forestiers est évident.

L'autre indice aussi important est sans doute l'accroissement moyen. La courbe de l'accroissement moyen $p(t)$ à la figure 1 est définie par l'équation suivante:

$$
p(t)=\frac{h(t)}{t}=\frac{A}{t} e^{\frac{k}{(1-n) t^{n-1}}}
$$

C'est une courbe asymétrique avec un maximum au temps

$$
t_{2}=k^{\frac{1}{n-1}} \text {, }
$$

qui correspond à la culmination de l'accroissement moyen, et souvent à l'âge de la révolution.

Les deux points d'inflexion de la courbe $p(t)$ se situent aux temps $t_{5}$ et $t_{6}$, ainsi

$$
t_{5}=\left[\frac{k}{4}\left(n+2-\left(n^{2}+4 n-4\right)^{\frac{1}{3}}\right)\right]^{\frac{1}{n-1}}
$$

$$
t_{6}=\left[\frac{k}{4}\left(n+2+\left(n^{2}+4 n-4\right)^{\frac{1}{3}}\right)\right]^{\frac{1}{n-1}}
$$

et l'asymptote de la courbe est l'axe de t.

L'examen de trois paramètres $k, n$ et $A$ de la fonction (5) nous permet d'établir que:

a) les paramètres $n$ et $k$ influencent la forme des courbes de la croissance et des accroissements mais aussi le temps de culmination des accroissements donc, ils reflètent les conditions de la croissance,

b) le paramètre $A=\lim _{t \rightarrow \infty} h$ 'a pas d'influence sur la forme des courbes, mais il influence directement la grandeur de la variable de croissance.

Notons finalement que la fonction de Korf, par l'asymétrie de ses courbes, satisfait la condition sine qua non de Backman (1943), pour une fonction de la croissance organique.

Application de la fonction de Korf à l'étude de la rela. tion hauteurlâge pour l'épinette noire sur trois sites de qualité différente.

Pour le besoin de notre exemple, nous avons utilisé les données sur la hauteur (mesurée en mètres au dixième près) et l'âge recueillies au cours de l'inventaire écologique du territoire de la Baie James en 1975, par l'équipe de la Direction régionale des Terres, Environnement Canada à Québec. L'essence considérée était l'épinette noire (Picea mariana Mill. B.S.P.).

Les caractéristiques stationnelles des trois sites ainsi que leur localisation sont résumées au tableau 1.

Pour estimer les paramètres de la fonction de Korf, nous

Y

A

$x^{2}+2$
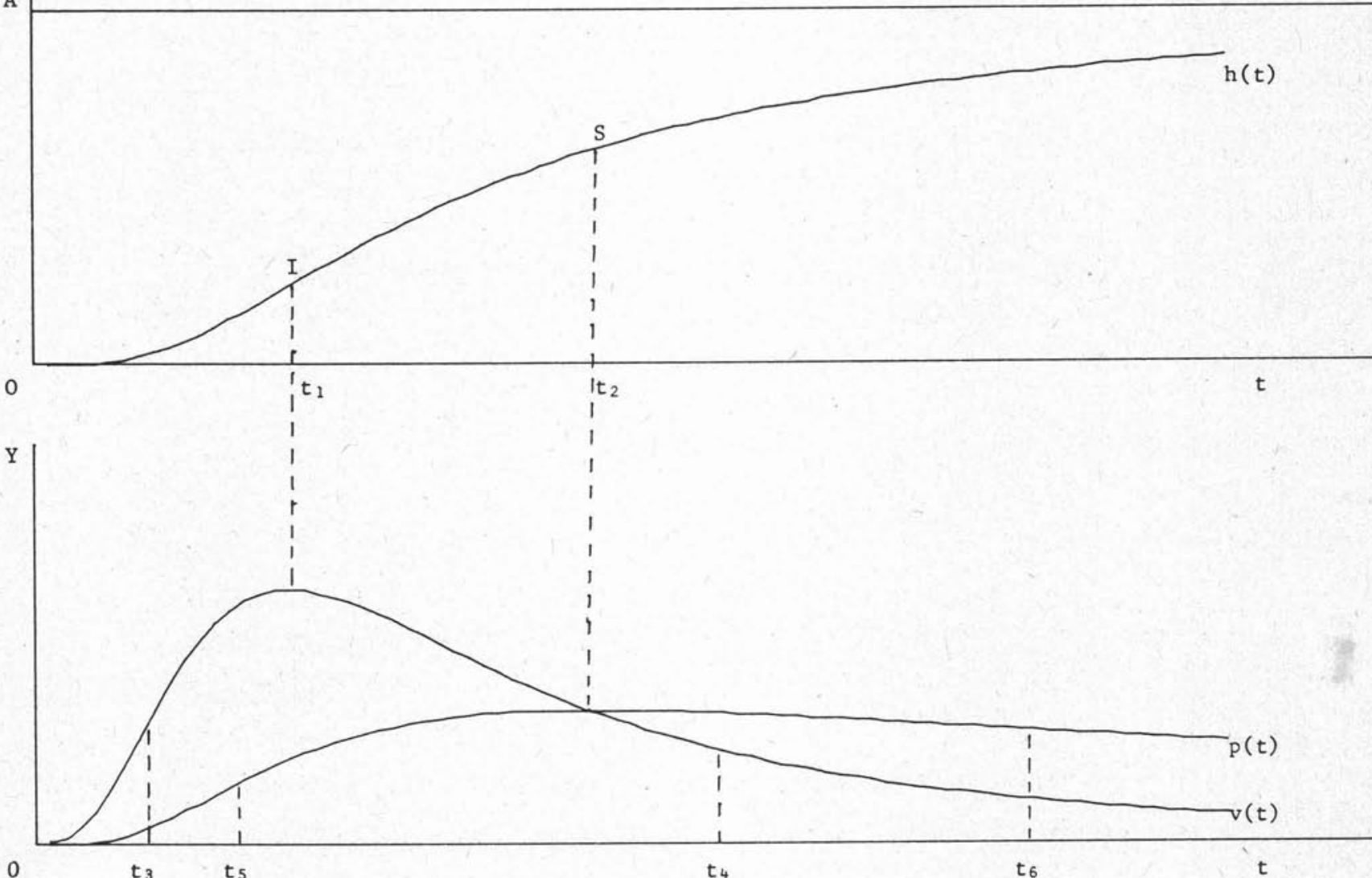

$t_{3} \quad t_{5}$

$t_{4}$

Fig 1: Expression graphique des courbes de la croissance et des accroissements. 
avons utilisé le programme "PAR" de BMDP "biomedical computer program"' implanté au CTI de I'Université Laval.

Les résultats de la régression concernant la relation hauteur/âge de l'épinette noire pour les trois sites sont résumés au tableau 2.

II est à remarquer que la fonction de Korf permet une estimation suffisamment précise de la hauteur des arbres d'épinette noire en fonction de l'âge pour les trois sites étudiés. D'ailleurs, l'écart type résiduel ainsi que le coeffi-

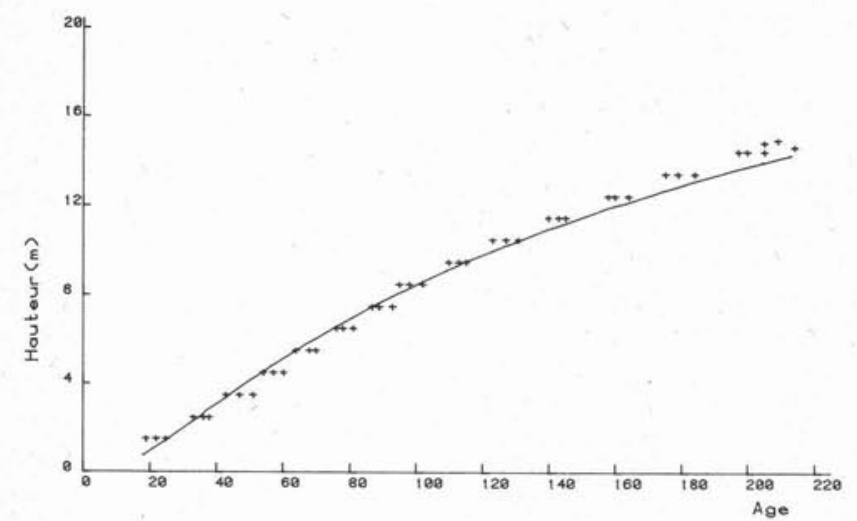

Fig. 2a: Hauteur en fonction de l'âge pour l'épinette noire sur le site 1.

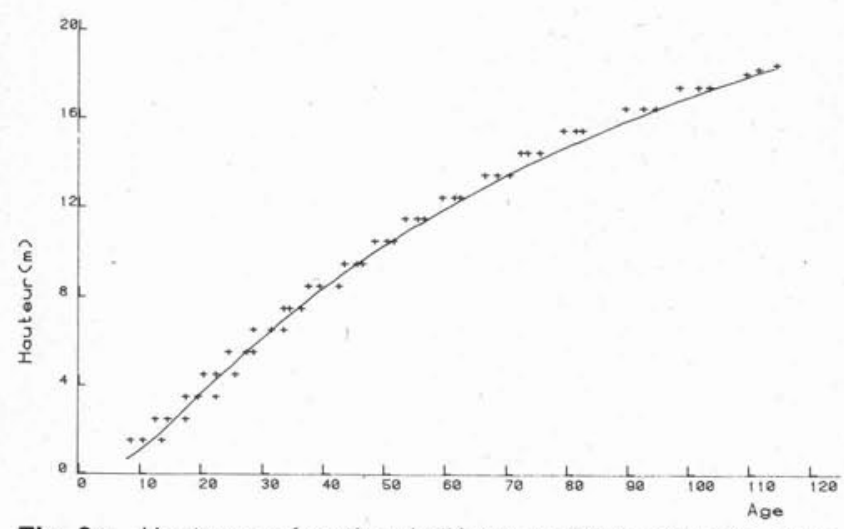

Fig. 3a: Hauteur en fonction de l'âge pour l'épinette noire sur le site 2. cient de détermination en sont la preuve (tableau 2).

En examinant les résultats sur l'âge de culmination des accroissements courant et moyen en hauteur des arbres de l'épinette noire (tableau 2), on peut dire que la phase de pleine vigueur atteint son maximum à 18 ans et qu'elle s'arrête à 39 ans pour le site 1 , tandis qu'elle culmine à 33 ans et ne s'arrête qu'à 74 ans pour le site 2 , et que finalement la croissance de pleine vigueur culmine à 63 ans et s'arrête à 139 ans sur le site 3 .

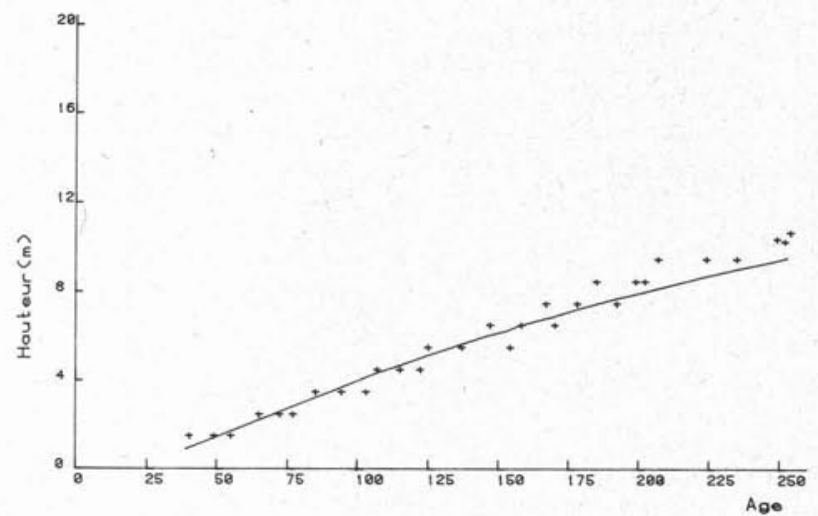

Fig. 4a: Hauteur en fonction de l'âge pour l'épinette noire sur le site 3.

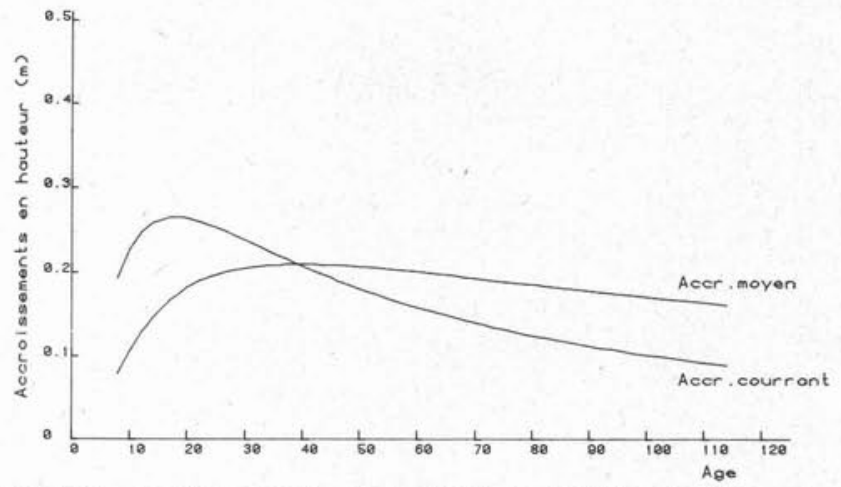

Fig.2b: Accroissements en hauteur en fonction de l'âge pour l'épinette noire sur le site 1.

\begin{tabular}{ccccccc}
\hline \multicolumn{7}{c}{ Tableau 1: Principales caractéristiques stationnelles des trois sites étudiés } \\
\hline Site & $\begin{array}{c}\text { Texture } \\
\text { du sol }\end{array}$ & $\begin{array}{c}\text { Drainage* } \\
\text { du sol }\end{array}$ & LAT. & Long. & $\begin{array}{c}\text { Alt. } \\
(\mathbf{m})\end{array}$ & Groupement végétal \\
\hline 1 & $\begin{array}{c}\text { loam } \\
\text { argileux } \\
\text { loam } \\
\text { argileux } \\
\text { limon } \\
\text { argileux }\end{array}$ & $2-3$ & $50^{\circ} 16^{\prime}$ & $78^{\circ} 50^{\prime}$ & 180 & Pessière noire à mousses \\
2 & 5 & $50^{\circ} 23^{\prime}$ & $78^{\circ} 25^{\prime}$ & 240 & $\begin{array}{l}\text { Pessière noire à mousses et } \\
\text { sphaignes }\end{array}$ \\
3 & 5 & $50^{\circ} 23^{\prime}$ & $78^{\circ} 25^{\prime}$ & 240 & $\begin{array}{l}\text { Pessière noire à sphaignes et } \\
\text { éricacées }\end{array}$ \\
\hline
\end{tabular}

*Selon la Commission canadienne de Pédologie, 1972.

Tableau 2: Principales caractéristiques de la fonction de Korf pour la relation hauteur/âge de l'épinette noire sur les trois sites étudiés.

\begin{tabular}{|c|c|c|c|c|c|c|c|c|c|c|c|}
\hline \multirow[t]{2}{*}{ Site } & \multirow{2}{*}{$\begin{array}{c}\text { Nombre } \\
\text { d'observations }\end{array}$} & \multirow{2}{*}{\multicolumn{2}{|c|}{$\begin{array}{c}\text { Paramètres } \\
\mathbf{k}\end{array}$}} & \multirow[b]{2}{*}{$\mathbf{n}$} & \multirow{2}{*}{\multicolumn{4}{|c|}{ 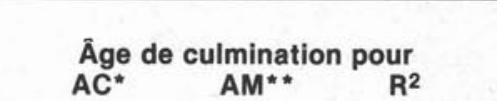 }} & \multirow{2}{*}{$\begin{array}{c}\text { Somme des } \\
\text { carrés } \\
\text { de résidus }\end{array}$} & \multicolumn{2}{|c|}{$\begin{array}{l}\text { Ecart type } \\
\text { résiduel }\end{array}$} \\
\hline & & & & & & & & & & en $m$ & en $\%$ \\
\hline 1 & 54 & 49,44 & 7,69341 & 1,55641 & 17,7 & $\div 18$ & $39,1-39$ & 0,994 & 8,272 & 0,03 & 1,5 \\
\hline 2 & 45 & 40,87 & 10,20000 & 1,53972 & 33,2 & -33 & $73,9 \div 74$ & 0,991 & 6,964 & 0,04 & 1,9 \\
\hline 3 & 30 & 35,03 & 15,20000 & 1,551724 & 62,6 & -63 & $138,7-139$ & 0,961 & 8,644 & 0,11 & 5,3 \\
\hline
\end{tabular}

*Accroissement courant

**Accroissement moyen 


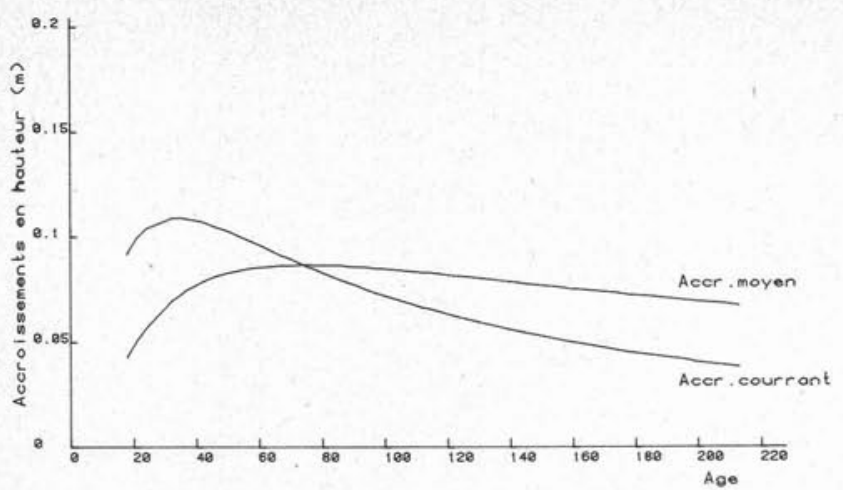

Fig. 3b: Accroissements en hauteur en fonction de l'âge pour l'épinette noire sur le site 2.

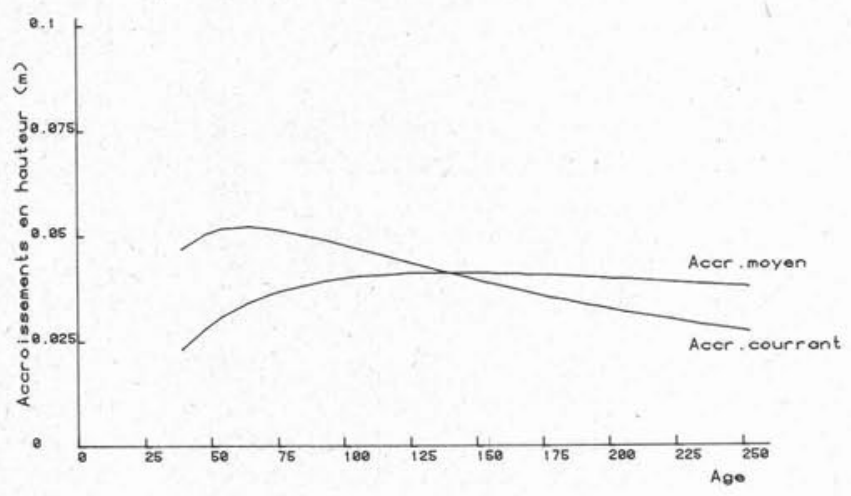

Fig. 4b: Accroissements en hauteur en fonction de l'âge pour l'épinette noire sur le site 3 .
Ceci nous permet d'évaluer la performance de trois sites relativement à la vitesse de croissance en hauteur des arbres d'épinette noire en fonction de l'âge. Ainsi, l'accroissement courant en hauteur des épinettes sur le site 1 culmine presque deux fois plus vite que celui du site 2 et plus que trois fois plus vite que celui du site 3 .

L'expression graphique des valeurs observées et calculées pour la hauteur de l'épinette noire en fonction de l'âge de trois sites respectifs se trouve sur les figures $2 a, 3 a$ et $4 \mathrm{a}$. Par contre, les valeurs calculées des accroissements courant et moyen en hauteur de l'épinette noire en fonction de l'âge pour trois sites étudiés sont exprimées graphiquement sur les figures $2 b, 3 b$ et $4 b$.

\section{Remerciements}

L'auteur désire remercier monsieur M. Fortin, professeur à l'Université Laval, qui a relu ce texte et y a apporté des suggestions fort pertinentes, et monsieur F. Bonenfant, Section BiométrieInformatique, CRFL. pour son aide lors du traitement des données.

\section{Bibliographie}

Backman, G. 1943. Wachstum und organische Zeit. Roux ArchivLeipzig.

Korf, V. 1967. Rust stejnovekych porostu a Backmanova rustova teorie. Les. cas., $13(10), 855-870$.

Korf, V. 1974. Diferencialni rovnice v rustové teorii. Sbornik CSAZV, 17, 3-21. 